\title{
Enhanced Microlensing by Stars around the Black Hole in the Galactic Center
}

\section{Citation}

Alexander, Tal, and Abraham Loeb. 2001. "Enhanced Microlensing by Stars around the Black Hole in the Galactic Center." The Astrophysical Journal 551 (1): 223-30. https:// doi.org/10.1086/320087.

\section{Permanent link}

http://nrs.harvard.edu/urn-3:HUL.InstRepos:41393286

\section{Terms of Use}

This article was downloaded from Harvard University's DASH repository, and is made available under the terms and conditions applicable to Other Posted Material, as set forth at http:// nrs.harvard.edu/urn-3:HUL.InstRepos:dash.current.terms-of-use\#LAA

\section{Share Your Story}

The Harvard community has made this article openly available.

Please share how this access benefits you. Submit a story.

Accessibility 


\title{
Enhanced Microlensing by Stars Around the Black Hole in the Galactic Center
}

\author{
Tal Alexander \\ Space Telescope Science Institute, 3700 San Martin Drive, Baltimore, MD 21218 \\ and
}

Abraham Loeb

Harvard-Smithsonian Center for Astrophysics, 60 Garden Street,Cambridge, MA 02138

The effect of stars on the lensing properties of the supermassive black hole in the Galactic Center is similar to the effect of planets on microlensing by a star. We show that the dense stellar cluster around $\mathrm{SgrA}^{\star}$ increases by factors of a few the probability of high-magnification lensing events of a distant background source by the black hole. Conversely, the gravitational shear of the black hole changes and enhances the microlensing properties of the individual stars. The effect is largest when the source image lies near the Einstein radius of the black hole $\left(1.75^{\prime \prime} \pm 0.20^{\prime \prime}\right.$ for a source at infinity). We estimate that the probability of observing at least one distant background star which is magnified by a factor $>5$ in any infrared snapshot of the inner $\sim 2$ " of the Galactic Center is $\sim 1 \%$ with a $K$-band detection threshold of 20 mag. The largest source of uncertainty in this estimate is the luminosity function of the background stars. The gravitational shear of the black hole lengthens the duration of high-magnification events near the Einstein radius up to a few months, and introduces a large variety of lightcurve shapes that are different from those of isolated microlenses. Identification of such events by image subtraction can be used to probe the mass function, density and velocity distributions of faint stars near the black hole, which are not detectable otherwise.

Subject headings: Galaxy: center — gravitational lensing — infrared: stars — Galaxy: stellar content

\section{Introduction}

Deep infrared observations of the innermost region around the massive black hole $(\mathrm{BH})$ in the Galactic Center (GC) reveal numerous point sources (Genzel et al. 1997; Ghez et al. 1998). Most of these are probably stars orbiting deep in the potential of the BH. However, the BH will also gravitationally lens and magnify any background star that happens to lie behind it, and so a small fraction of these point sources could be lensed images of distant background stars. The possible existence of such images in the innermost GC, beyond being a probe of the BH potential, may also affect the apparent star counts, radial stellar density distribution and infrared luminosity function. Previous investigations of gravitational lensing by the BH (Wardle \& Yusef-Zadeh 1992; Alexander \& Sternberg 1999) suggested that lensing effects do not play a major role in present-day observations of the GC. However, the BH is surrounded by a very dense stellar cluster (e.g. Genzel 
et al. 1997), whose contribution to the lensing by the $\mathrm{BH}$ has so far been neglected. Because the stellar mass is not smoothly distributed around the BH but is composed of discrete point masses, its effect on the lensing properties of the $\mathrm{BH}$ is much larger than one may naively estimate by adding the stellar mass to that of the $\mathrm{BH}$.

The effect of a star on lensing by the $\mathrm{BH}$ is analogous to the effect of a planet on microlensing by its star. The latter problem has been studied in great detail in the context of microlensing searches for planets (Mao \& Paczynski 1991; Gould \& Loeb 1992; Bolatto \& Falco 1994; Gaudi \& Gould 1997; Wambsganss 1997; Peale 1997; Griest \& Safizadeh 1998; Gaudi, Naber, \& Sackett 1998; Gaudi \& Sackett 1997). In particular, Gould \& Loeb (1992) first showed that the cross-section for magnification by the planet can be increased by up to an order of magnitude due to the gravitational shear of the star; the effect being most pronounced when the planet lies near the Einstein radius of the star. Aside from the change in scales, the same result should apply to $\mathrm{Sgr}^{\star}$. In the GC, the primary massive lens is the $\mathrm{BH}$ and the secondary low-mass lenses are the stars around it. If a star happens to pass near the image of a source that is being lensed by the $\mathrm{BH}$, then the magnification of the image could be enhanced significantly. The BH shear increases the probability for high magnification events by individual stars. Miralda-Escudé \& Gould (2000) pointed out that the existence of a cluster of stellar mass BHs around the massive $\mathrm{BH}$ could be detected by this effect. In this paper we consider normal stellar lenses and calculate the probability of high-magnification events in the stars-BH system in comparison to the naked $\mathrm{BH}$ case.

The statistics of microlensing by stars in the GC region is very different from that in the Galactic disk or halo. In the latter case, the optical depth for microlensing is very small, $\tau \sim 10^{-7}-10^{-5}$, but there are many background sources (see, e.g. review by Paczynski 1996). In contrast, the optical depth for microlensing by stars within the inner arcsecond of the GC is as high as $\sim 0.1$ due to the high stellar density there (see $\S$ ) , but it is not clear whether there is a sufficient number of bright background sources. In fact, due to their large distances and high extinction by dust, most sources behind the GC will only be observable while being magnified during a lensing event. The microlensing events are transient; any distant source behind the GC will be microlensed for a fraction of the time due to foreground stars which are passing in front of its images.

This paper is organized as follows. The method of our calculation is described in 8 2; the models of the stellar lenses and background stellar sources are described in $\oint 3$; and the numerical results are presented in $\$$. We discuss the main implications of our results in $\$$.

\section{Method}

We adopt the semi-analytic method of Gould \& Loeb 1992; see also the original discussion by Chang \& Refsdal 1979, 1984) to describe the effect of a low-mass secondary lens (a star) on the image of a point source produced by a high-mass primary lens (the $\mathrm{BH}$ ). The limitations of 
the point source assumption are discussed in 过. We consider rare, high-magnification events for which the possibility that more than one star affects the microlensing event at any given time can be neglected. The original, unperturbed image position at $\boldsymbol{x}_{i}$ is displaced by $\boldsymbol{\xi}_{i}=\left(\xi_{i}, \eta_{i}\right)$ due to the perturbing star at a position $\boldsymbol{\xi}_{p}=\left(\xi_{p}, \eta_{p}\right)$ relative to the unperturbed image (Fig. 1). We express the source position $\boldsymbol{x}_{s}$ and the unperturbed image position $\boldsymbol{x}_{i}$ in terms of the Einstein angular radius of the isolated $\mathrm{BH}$,

$$
\theta_{E} \equiv\left[\frac{4 G M_{\bullet}}{c^{2}} \frac{\left(D_{s}-R_{0}\right)}{D_{s} R_{0}}\right]^{1 / 2}=\left(1-\frac{R_{0}}{D_{s}}\right)^{1 / 2} \theta_{\infty}
$$

where $M_{\bullet}=(3.0 \pm 0.5) \times 10^{6} M_{\odot}$ (Genzel et al. 2000) is the BH mass, $R_{0}=8.0 \pm 0.5 \mathrm{kpc}$ (Reid 1993) is the distance to the GC, $D_{s}$ is the distance to the source, and $\theta_{\infty}=1.75^{\prime \prime} \pm 0.20^{\prime \prime}$ is the Einstein angle for a source at infinity $\left(1^{\prime \prime} \simeq 0.04 \mathrm{pc}\right.$ at the GC). We express the displacement vectors $\boldsymbol{\xi}_{i}$ and $\boldsymbol{\xi}_{p}$ in terms of the Einstein radius of the star, $\sqrt{\epsilon} \theta_{E}$, where $\epsilon=m_{\star} / M_{\bullet}$ is the mass ratio between the star and the $\mathrm{BH}$. This quantity provides a good measure for the scale of the stellar lensing zone. Correspondingly, we express areas in the lens plane, $\sigma_{\star}$, in units of $\epsilon \pi \theta_{E}^{2}$, the Einstein ring area of an isolated star; and express surface number densities of lensing stars in the lens plane, $\Sigma_{\star}$, by $\left(\epsilon \pi \theta_{E}^{2}\right)^{-1}$. Areas in the source plane, $\sigma_{s}$, are expressed in terms of $\pi \theta_{E}^{2}$, and surface number density of sources in the source plane, $\Sigma_{s}$, are correspondingly expressed in terms of $\left(\pi \theta_{E}^{2}\right)^{-1}$.

In the limit $\epsilon \ll 1, \xi_{i}$ is given by the two or four solutions to the equations (Gould \& Loeb 1992)

$$
\begin{aligned}
\xi_{i}^{4}+\frac{(1-2 \gamma) \xi_{p}}{\gamma} \xi_{i}^{3}+\left[\frac{(1-\gamma)^{2}\left(\xi_{p}^{2}+\eta_{p}^{2}\right)}{4 \gamma^{2}}-\frac{(1-\gamma) \xi_{p}^{2}}{\gamma}-\frac{1}{1+\gamma}\right] \xi_{i}^{2}- \\
{\left[\frac{(1-\gamma)^{2}\left(\xi_{p}^{2}+\eta_{p}^{2}\right) \xi_{p}}{4 \gamma^{2}}+\frac{(1-\gamma) \xi_{p}}{\gamma(1+\gamma)}\right] \xi_{i}-\frac{(1-\gamma)^{2} \xi_{p}^{2}}{4 \gamma^{2}(1+\gamma)}=0 }
\end{aligned}
$$

where $\gamma \equiv x_{i \bullet}^{-2}$, and

$$
\eta_{i}=\frac{(1+\gamma) \eta_{p} \xi_{i}}{2 \gamma \xi_{i}+(1-\gamma) \xi_{p}}
$$

The magnification of each of the images is

$$
A=\left|1-\left[\gamma+(1+\gamma)^{2} \xi_{i}^{2}-(1-\gamma)^{2} \eta_{i}^{2}\right]^{2}-4\left(1-\gamma^{2}\right)^{2} \xi_{i}^{2} \eta_{i}^{2}\right|^{-1}
$$

For the GC, we consider solar mass lenses and adopt, for simplicity, a single value of $\epsilon=3 \times 10^{-7}$.

The angular area in the source plane where a background source would be magnified above a given threshold $A$ by the star-BH system, $\sigma_{s}(>A)$, is obtained by identifying the corresponding angular area in the lens plane around the unperturbed image and transforming it back to the source plane円. The lensing stars are distributed randomly around the BH and they scan the lens

\footnotetext{
${ }^{1}$ Source areas that contribute two images which are above the magnification threshold, are counted twice.
} 
plane as they move. Averaging over time and assuming an isotropic projected stellar distribution around the $\mathrm{BH}$,

$$
\sigma_{s}(>A)=\frac{1}{\pi} \int P\left(>A, \boldsymbol{x}_{i \bullet}\right)\left|\frac{d \boldsymbol{x}_{s}}{d \boldsymbol{x}_{i \bullet}}\right| d \boldsymbol{x}_{i \bullet}=2 \int P\left(>A, \boldsymbol{x}_{i \bullet}\right) A_{\bullet}^{-1}\left(x_{i \bullet}\right) x_{i \bullet} d x_{i \bullet}
$$

where $P\left(>A, \boldsymbol{x}_{i \bullet}\right)$ is the probability for magnification above $A$, that is, the fraction of $d \boldsymbol{x}_{i \bullet}$ where the image is magnified above $A$. Equivalently, $P\left(>A, \boldsymbol{x}_{i \bullet}\right)$ is the fraction of time a stationary source is magnified above $A$ by a closely-passing star. The magnification $A \bullet$ is that due to the $\mathrm{BH}$ alone,

$$
A_{\bullet}=\left|\frac{d \boldsymbol{x}_{i \bullet}}{d \boldsymbol{x}_{s}}\right|=\left|1-x_{i \bullet}^{-4}\right|^{-1},
$$

where the positions of these images are related to the source position,

$$
x_{i \bullet, \pm}=\frac{1}{2}\left(x_{s} \pm \sqrt{x_{s}^{2}+4}\right) .
$$

De-magnification, i.e. $A<A_{\bullet}$, is also possible. The probability $P$ is expressed in terms of the optical depth

$$
\tau_{\star}\left(>A, x_{i \bullet}\right) \equiv \Sigma_{\star}\left(x_{i \bullet}\right) \sigma_{\star}\left(>A, x_{i \bullet}\right),
$$

where $\sigma_{\star}\left(>A, x_{i} \bullet\right)$ is the area in the lens plane where the presence of a star will lead to magnification above $A$, and $\Sigma_{\star}\left(x_{i \bullet}\right)$ is the stellar lens surface density. In the small optical depth limit

$$
P\left(>A, \boldsymbol{x}_{i \bullet}\right) \simeq \tau_{\star}\left(>A, x_{i \bullet}\right)+\Theta(A \bullet-A),
$$

where $\Theta$ is the Heaviside step function. In practice, the lens plane area $\sigma_{\star}\left(>A, x_{i \bullet}\right)$ is calculated by solving equations (2)-(勾) numerically as functions of $\boldsymbol{\xi}_{p}$ in a small test area $\sigma_{0}\left(x_{i \bullet}\right)$ around $\boldsymbol{x}_{i \bullet}$, where the effect of the stellar lens magnification is appreciable. The effect of the star outside of $\sigma_{0}$ is neglected. With this assumption, the probability for not being affected by any star is $\exp \left(-\tau_{0}\right)$, where $\tau_{0} \equiv \Sigma_{\star} \sigma_{0}$. The magnification probability is then given by the sum of the probabilities of magnification by a star or by the BH alone, $P(>A)=\left[1-\exp \left(-\tau_{\star}\right)\right]+\exp \left(-\tau_{0}\right) \Theta\left(A_{\bullet}-A\right)$, which in the limit $\tau_{\star}, \tau_{0} \ll 1$ reduces to equation (9), independently of the exact choice of $\sigma_{0}$.

For an isolated $\mathrm{BH}\left(\Sigma_{\star}=0\right)$, the corresponding angular area in the high magnification limit, $A \gg 1$, is $\sigma_{s}(>A) \bullet \approx 1 / 2 A^{2}$.

Equations (2)-(目) are valid as long as $\tau_{\star}\left(>A, x_{i \bullet}\right) \ll 1$, i.e. when $A$ is large enough so that there is no overlap between the regions of influence (lensing zones) of different stars, $\sigma_{\star}\left(>A, x_{i}\right) \ll \Sigma_{\star}^{-1}\left(x_{i \bullet}\right)$. In addition, these equations ignore the cumulative effect of all the stars on the central caustic around the BH (Griest \& Safizadeh 1998). For $\epsilon=3 \times 10^{-7}$, the central caustic has a negligible contribution to the integrand of equation (5) as long as $x_{i}$ is not very close to unity (i.e. for sources which are not located almost behind the BH). The inclusion of the central caustic can only increase our estimated lensing probabilities. 


\section{Model}

In $\S$ 团 below we demonstrate the effect of enhanced microlensing by stars near a massive BH by calculating the mean number of lensed images with magnification above a threshold $A$, $\left\langle N_{i}(>A)\right\rangle=\sigma_{s}(>A) \Sigma_{s}$, of distant background sources behind $\operatorname{Sgr} A^{\star}$. To do this, we need to specify the projected density of the stellar lenses $\Sigma_{\star}$, which determines the optical depth for microlensing and hence the cross-section in the source plane $\sigma_{s}(>A)$ (eq. [5]), and we also need to specify the projected source density $\Sigma_{s}$ and source luminosity function. We express these surface densities scaled to $\theta_{\infty}=1.75^{\prime \prime}$.

\subsection{Stellar Lenses Around SgrA*}

The stellar mass density distribution near the $\mathrm{BH}$ is modeled as a power-law,

$$
\rho=(3-\alpha) \rho_{b}\left(\frac{r}{r_{b}}\right)^{-\alpha}
$$

with $\rho_{b}=10^{6} M_{\odot} \mathrm{pc}^{-3}, r_{b}=0.4 \mathrm{pc}$ and an index $\alpha$ in the range of $3 / 2$ to $7 / 4$. The normalization of the mass density is based on the dynamic mass measurements of Eckart \& Genzel (1997). The power-law distribution is indicated by an analysis of the star counts in the inner GC (Alexander 1999) and agrees with the theoretical prediction for a relaxed stellar system around a black hole (Bahcall \& Wolf 1977), as is thought to be the case in the GC. For simplicity, we assume that the stellar lenses all have the same mass $m_{\star}=1 M_{\odot}\left(\epsilon \approx 3 \times 10^{-7}\right)$. For these low mass stars, we adopt the theoretical prediction of $\alpha \sim 3 / 2$. The corresponding surface mass density (in units of $M_{\bullet} / \pi \theta_{E}^{2}$ ) and surface number density (in units of $1 / \epsilon \pi \theta_{E}^{2}$ ) at an angular separation $x=\theta / \theta_{E}$ from the GC are both given by

$$
\Sigma_{\star}=\widehat{\Sigma}_{\star} x^{1-\alpha}
$$

with $\widehat{\Sigma}_{\star} \simeq 0.04\left(\theta_{E} / \theta_{\infty}\right)^{3-\alpha}$. The normalization $\widehat{\Sigma}_{\star}$ is almost independent of $\alpha$ in the above range and scales as $M_{\bullet}^{-1}$. The ratio of stellar mass enclosed within the Einstein radius and the mass of the $\mathrm{BH}$ is $2 \widehat{\Sigma}_{\star} /(3-\alpha)$. Note that $\Sigma_{\star}$ is also the optical depth for lensing by the stars alone, neglecting the effect of the $\mathrm{BH}$. As we show below, the actual optical depth increases substantially when the shear of the $\mathrm{BH}$ is included, an effect analogous to the cross-section enhancement for planetary systems (Gould \& Loeb 1992).

\subsection{Distant Background Stars}

A rough estimate of the projected density of distant stars, which is based on a model of the $K$-band luminosity distribution in the Galaxy (Kent 1992) and the assumption that the mean stellar $K$-band luminosity is solar, suggests that there are of order $\sim 100$ distant background stars within $\sim 2^{\prime \prime}$ of the BH. We define "distant background stars" as those stars with $\theta_{E} \gtrsim 1^{\prime \prime}$, 
i.e. farther than $\sim 4 \mathrm{kpc}$ behind the $\mathrm{BH}$. The surface density of nearby background stars in the inner bulge and in the high density central cluster may be as high as $\sim 10^{3} \operatorname{arcsec}^{-2}$ (Alexander \& Sternberg 1999). However, the Einstein angular radius for stars so close behind the BH is less than $0.1^{\prime \prime}$ and there are not enough lensing stars near the $\mathrm{BH}$ on that scale for microlensing to become important. Here, we study the role of these close stars as microlenses rather than as sources.

The stellar $K$ luminosity function (KLF) of the distant background stars can be determined from observations in the $b=0^{\circ}, l=30^{\circ}$ direction (i.e. impact parameter of $4 \mathrm{kpc}$ relative to the GC). The infrared Galactic model of Ortiz \& Lépine (1993), which reproduces such observations, predicts $\Sigma_{s}\left(<13.5^{\mathrm{m}}\right) \sim 0.1$ (see their Fig. 17b) with a steep slope of $b \equiv d \log \Sigma_{s} / d K \sim 0.4$ to 0.5. The projected stellar population is integrated over most of the Galactic disk and is composed of stars of different ages, and so it is reasonable to approximate it as an old, continuously star forming population. Observations of such populations in the Galactic Bulge (Tiede et al. 1995; Holtzman et al. 1998) and the central cluster in the GC (Blum, Sellgren \& DePoy 1996; Davidge et al. 1997), as well as stellar population synthesis of the GC (Alexander \& Sternberg 1999), indicate that the post-main-sequence KLF, where the $K$-luminous sources lie, typically takes the form of a single power-law with $b \sim 0.3-0.4$ down to the main sequence turn-off point at around $M_{K \odot}=3.3^{\mathrm{m}}$, where the power-law flattens and then turns over. Assuming a typical distance of $2 R_{0}$ to the distant background sources and $A_{K} \sim 3^{\mathrm{m}}$ to the GC (Blum et al. 1996), and using the population synthesis models to estimate the mean stellar $K$ luminosity and the fraction of stars less massive than solar, we approximate the KLF as a single power law that extends down to a cutoff magnitude of $K_{c} \sim 28.2 \mathrm{mag}$ and normalize it by Kent's integrated luminosity density model. The uncertainty in the exact shape of the luminosity function, the patchiness of the extinction in the Galactic Plane, and the distribution of star-forming regions in the spiral arms behind the GC introduce a large uncertainty to $\Sigma_{s}$. Putting these uncertainties aside, we adopt a working model for the KLF of the distant background stars,

$$
\Sigma_{s}(<K)=\widehat{\Sigma}_{s} 10^{b K},
$$

with $\widehat{\Sigma}_{s}=5 \times 10^{-10}\left(\theta_{E} / \theta_{\infty}\right)^{2}$ and $b=0.4$.

The uncertainties inherent in the estimate of $\widehat{\Sigma}_{s}$ are demonstrated by the attempt to extrapolate to low luminosity the observed KLF and correct it for distance, projection, foreground stars and the higher extinction at $l=0^{\circ}$. The Galactic extinction model of Wainscoat et al. (1992) predicts that the integrated $K$-band extinction in the direction of the GC is $\sim 2^{\mathrm{m}}$ larger than that at $l=30^{\circ}$. The correction for foreground stars and for the projection from $l=30^{\circ}$ to $l=0^{\circ}$ (assuming an exponential Galactic disk with a length scale of $3.5 \mathrm{kpc}$, Wainscoat et al. 1992) further reduces the projected density by a factor of $\sim 0.5$, and the shift from a typical distance of $\sim R_{0}$ to $2 R_{0}$ by another factor of $\sim 0.25$. This gives $\Sigma_{s}\left(<15.5^{\mathrm{m}}\right) \sim 0.01\left(\theta_{E} / \theta_{\infty}\right)^{2}$ for the distant background stars, which corresponds to $\widehat{\Sigma}_{s}=5 \times 10^{-9}\left(\theta_{E} / \theta_{\infty}\right)^{2}, 10$ times higher than our adopted normalization (eq. 12).

The differential surface density of lensed stars, $d \widetilde{\Sigma}_{s} /\left.d \widetilde{F}\right|_{\widetilde{F}}$, is related to their unlensed 
luminosity function by $d \widetilde{\Sigma}_{s} /\left.d \widetilde{F}\right|_{\widetilde{F}}=A^{-2} d \Sigma_{s} /\left.d F\right|_{\widetilde{F} / A}$, where the tilde denotes lensed quantities and where $d \Sigma_{s} /\left.d F\right|_{F} \propto F^{-2.5 b-1} \propto F^{-2}$ for the KLF of equation (12). Hence, the adopted surface density has the critical power-law slope for which the differential flux distribution remains unchanged.

\section{Results}

Figure 2a shows the integrated source plane area $\sigma_{s}(>A)$ for producing a lensed image of a point source that is magnified by more than $A$ anywhere in the inner $2 \theta_{E}$, for $\theta_{E}=\theta_{\infty}$ and the stellar lens surface density model of equation (11) with $\alpha=3 / 2$. The results can be scaled to any value of $\theta_{E}$ or any stellar lens surface density normalization $\widehat{\Sigma}_{\star}$ by noting that $\sigma_{s}(>A)-\sigma_{s}(>A) \bullet \propto \widehat{\Sigma}_{\star} \propto \theta_{E}^{3-\alpha}$ (Eqs. [5], [9] and [11]). In particular, for $D_{s}=2 R_{0}$ the difference in source plane areas is decreased by a factor of 0.6 . The highest magnifications are due to stellar lenses close to $\theta_{E}$. The integration range in equation (5) does not include the annulus $\left|1-x_{i \bullet}\right|<\delta x=0.005$. At that point the semi-analytic approximation (eq. [2]) and the small optical depth assumption no longer apply because the change in $A_{\bullet}$ over the angular scale defined by $\sigma_{\star}\left(>A, x_{i}\right)$ is no longer small, and $\tau_{\star}\left(>A, x_{i \bullet}\right) \ll 1$ no longer holds even for very high values of $A$. We verified the validity of our semi-analytic calculations for the case of a $\mathrm{BH}$ and a single star in the range $\delta x>0.001$ by comparing $\sigma_{\star}\left(>A, x_{i \bullet}\right)$ as function of $A$ and $x_{i}$, with results from the direct ray shooting method (Wambsganss \& Kubas 2000). The two methods give identical results to within $\sim 5 \%$ down to $\delta x=0.1$ for both the normalization and slope and remain in good qualitative agreement even down to $\delta x=0.001$ throughout the range where the ray-shooting method has sufficiently high resolution.

For a star at a particular location, the magnification probability approaches the asymptotic $A^{-2}$ behavior in accordance with the theoretical asymptotic limit for a point source (eq. [11.21b] of Schneider, Ehlers \& Falco 1992) at some minimum magnification $A_{b}$, which depends on the external shear at that location. As the projected location of the image approaches $\theta_{E}$, the $A^{-2}$ tail is recovered at an increasingly larger value of $A_{b}$. With the above choice for $\delta x$, we find that the area $\sigma_{s}(>A)$ initially falls off as $A^{-3 / 2}$ up to a break at $A_{b} \gtrsim 50$ where it begins to converge to the asymptotic $A^{-2}$ behavior. In our semi-analytic approach, the value of $A_{b}$ depends on the choice of the truncation radius $\delta x$, with smaller values of $\delta x$ corresponding to larger values of $A_{b}$. An exact ray-shooting calculation is required to determine $A_{b}$ more precisely for a point source. Nevertheless, we note that our calculated value for $\sigma_{s}(>A)$ in the asymptotic limit agrees very well with the analytic expression for a binary lens (eq. [11.23a] of Schneider, Ehlers \& Falco 1992), taking into account the fact that our definition of $\gamma$ coincides with the shear parameter in the limit $\varepsilon \rightarrow 0$ (Dominik 1999, Eqs. [12] and [66])

$$
\sigma_{s}(>A \gg 1)=\left[\frac{4}{\pi} \widehat{\Sigma}_{\star} \int x_{i \bullet}^{2-\alpha} \frac{E(2 \sqrt{\gamma} /[1+\gamma])}{\left|1-\gamma^{2}\right||1-\gamma|} d x_{i}\right] \frac{1}{A^{2}} \simeq 66 \frac{\widehat{\Sigma}_{\star}}{A^{2}},
$$


where the integral was evaluated for $\alpha=3 / 2$ on the interval $\left(0 \leq x_{i} \bullet 1-\delta x\right) \cup\left(1+\delta x<x_{i} \leq 2\right)$ and where $E$ is the complete elliptic integral of the 2nd kind.

At high magnification the extended nature of the stellar sources can no longer be neglected. The maximal magnification for an extended source of angular size $\theta_{s}$ is of order $A_{\max } \sim \sqrt{\epsilon^{1 / 2} \theta_{E} / \theta_{s}}$ (Schneider, Ehlers \& Falco 1992, p. 215), which for a $1 R_{\odot}$ star located at $2 R_{0}$ gives $A_{\max } \sim 60$ and for a $10 R_{\odot}$ giant at the same location gives $A_{\max } \sim 20$. The absence of the high-magnification events is compensated by an increase in $\sigma_{s}$ below $A_{\max }$, which is not taken into account here. We find that even for magnifications $A \ll A_{\max }$, the cross-section of the $\mathrm{BH}$ and stars system is larger by factors of $\sim 2$ for sources at $D_{s}=2 R_{0}$, depending on the normalization of the surface density model for the lensing stars.

Figure 2 $2 \mathrm{~b}$ shows the differential area $\mathrm{d} \sigma_{s}(>A) / \mathrm{d} x_{i \bullet}$, for the $\mathrm{BH}$ with and without the stars. The contribution of stars to the high magnification events extends well beyond the narrow region around the Einstein radius that is expected for an isolated $\mathrm{BH}$.

Figure 3a shows the number of lensed images that will be observed, on average, in the inner $2 \theta_{E}$ for different limiting $K$-magnitudes, for $D_{s}=2 R_{0}$ and $\alpha=3 / 2$

$$
\begin{aligned}
\left\langle N_{i}\left(>A ; K_{0}\right)\right\rangle & =\left.\left.\int_{A}^{\infty} d A^{\prime} \int_{-\infty}^{K_{0}} d K \frac{d \sigma_{s}}{d A}\right|_{A^{\prime}} \frac{d \Sigma_{s}}{d K}\right|_{K+K_{A^{\prime}}} \\
& =\widehat{\Sigma}_{s} \widehat{\sigma}_{s}\left\{\begin{array}{cc}
10^{b K_{c}} A_{c}^{-1.5}+\frac{3}{5 b-3} 10^{b K_{0}}\left(A_{c}^{2.5 b-1.5}-A^{2.5 b-1.5}\right) & \text { if } A \leq A_{c} \\
10^{b K_{c}} A^{-1.5} & \text { else }
\end{array},\right.
\end{aligned}
$$

where we assume that the KLF of equation (12) has a sharp cutoff beyond $K_{c}$, and where $K_{A} \equiv 2.5 \log A, A_{c} \equiv 10^{0.4\left(K_{c}-K_{0}\right)}$, and $\sigma_{s}$ is parameterized as $\sigma_{s}(>A)=\widehat{\sigma}_{s} A^{-1.5}$ with $\widehat{\sigma}_{s} \approx 0.16$ (Fig. 2a). An analogous expression with $A^{-2}$ and a lower normalization describes the magnification by the $\mathrm{BH}$ alone. For low values of $A$ and $K_{0},\left\langle N_{i}\left(>A ; K_{0}\right)\right\rangle$ decreases slowly like $\propto A^{2.5 b-1.5}$ because the decrease of $\sigma_{s}(>A)$ is partially offset by the ever larger number of faint stars in the population that become accessible. However, once $K_{0}+K_{A}>K_{c}$, all the background stellar population can be observed when magnified by $A$, and $\left\langle N_{i}\left(>A ; K_{0}\right)\right\rangle$ falls off more rapidly, like $\sigma_{s}(>A) \propto A^{-1.5}$.

The motions of the stellar lenses and the sources introduce time-dependent Poissonian fluctuations in $N_{i}\left(>A ; K_{0}\right)$. The fraction of time that at least one lensed image will be observed, which is shown in Fig. [3b, is $P\left[N_{i}\left(>A ; K_{0}\right) \geq 1\right]=1-\exp \left[-\left\langle N_{i}\left(>A ; K_{0}\right)\right\rangle\right]$.

Present-day observations reach a depth of $K_{0} \sim 16^{\mathrm{m}}$ (Ghez et al. 1998). For this threshold, the probability of seeing at least one distant background star in the inner $2 \theta_{E} \sim 2^{\prime \prime}\left(D_{s}=2 R_{0}\right)$ with $A>5$ is small, $\sim 0.02 \%$. The lensing statistics will improve significantly with deeper observations. We predict that in any snapshot of the central $2^{\prime \prime}$ region around the GC to a $K$-magnitude limit of $23^{\mathrm{m}}$, at least one distant background star will be magnified by a factor $>5$ for $10 \%$ of the time.

Variability offers a direct way of identifying the lensed images among the many foreground 
stars. Any source detected with a magnitude $K_{0}$ at a given position $x_{i} \bullet$ will be magnified by an additional factor of $\geq A$ for a fraction $\sim 1 / A^{2}$ of the time (Schneider et al. 1992). Hence, continuous monitoring of detected sources would allow identification of microlensing events. Such events can be distinguished from variability of the source stars or from variable patchiness in the dust obscuration, through their achromaticity and their generic set of possible time profiles. The determination of the microlensing probability and duration distribution can be used to constrain the density and velocity distribution of low-mass stars in the GC. We demonstrate this connection qualitatively by defining a typical timescale for magnification by more than $A$ as

$t(>A) \equiv \sqrt{\sigma_{\star}(>A)} / v_{\perp} \propto \sqrt{m_{\star}} / v_{\perp}$ (Although the actual event duration depends on the complex caustics geometry, we use this definition as a crude estimate). The projected rms transverse velocity of the low mass stellar lenses in the GC is (Alexander 1999)

$$
v_{\perp}^{2} \approx 0.37 \frac{G M_{\bullet}}{p}
$$

where $p=\theta R_{0}$ is the projected distance from the BH. Figure 1 compares $t(>A)$ of magnified images of stationary sources lensed by solar mass stars in the shear field of SgrA ${ }^{\star}$ with the timescale of lensing by isolated stars (with the same velocity field). The duration of a microlensing event strongly depend on the position of the image, and is increased from several weeks to several months near $\theta_{E}$. The formal divergence of $t(>A)$ near $\theta_{E}$ is truncated in practice by the rapidly decreasing probability of having a source with an image close to $\theta_{E}$ (cf Fig. 2b) and by the motion of the source, which is neglected here. Nevertheless, a sharp increase of the microlensing timescales is expected near $\theta_{E}$. In contrast with timescales of lensing by isolated stars, the timescales inside $\theta_{E}$ fall rapidly to zero in the presence of the $\mathrm{BH}$ because the strong de-magnification by the $\mathrm{BH}$ (eq. [7]) has to be compensated by very high stellar lens magnification. The lensed lightcurves should show complex structures, unlike the symmetric smooth lightcurves of an isolated point mass lens. Examples of such lightcurves can be found in the literature (Mao \& Paczynski 1991; Gould \& Loeb 1992; Bolatto \& Falco 1994, and in particular Wambsganss 1997).

\section{Discussion and Summary}

The presence of a dense stellar cluster around a super-massive $\mathrm{BH}$ changes considerably the lensing properties of both the stars and the $\mathrm{BH}$ : the magnification cross-section, the magnification probability, the variability timescale and the lightcurve structure. The modification of the microlensing properties of the stars is most pronounced near the Einstein radius of the BH. Previous work on lensing by the BH alone (Wardle \& Yusef-Zadeh 1992; Alexander \& Sternberg 1999) focused on stellar sources from the inner central cluster, for which $\theta_{E} \sim 0.02^{\prime \prime}$. Here we have considered lensing of distant background stars by the $\mathrm{BH}$ and the stars around it, for which the angular scale is $\theta_{E} \sim 2^{\prime \prime}$.

We applied our calculations to the $\mathrm{BH}$ and the dense central cluster in the GC, and found that the lensing probability is increased over that of an isolated $\mathrm{BH}$ by factors ranging from $\sim 2$ 
for low magnifications $(\gtrsim 5)$ to $\sim 3$ for high magnifications $(\sim 50)$. We estimate that in any snapshot of the central $2^{\prime \prime}$ region around the GC to a $K$-magnitude limit of $23^{\mathrm{m}}$, at least one distant background star will be magnified by a factor $z 5$ for $10 \%$ of the time. The duration of a microlensing event can be lengthened by an order of magnitude near the Einstein radius of the BH. Moreover, the gravitational shear of the BH changes considerably the shape of the microlensing lightcurves so that they are no longer symmetric and smooth as they are for isolated stars.

Variability provides the distinguishing signature of microlensed stars. Image subtraction can be used to pick out lensed images of distant background sources from the many foreground stars. The achromaticity of the lightcurves and their generic (non-periodic) temporal structures can be used to separate them from those of intrinsically variable stars. Since the variability timescale scales as $\sqrt{m_{\star}} / v_{\perp}$, the lightcurves contain information on the stellar mass function, the stellar density, and the stellar velocity dispersion as functions of the projected distance from the BH. In particular, the lensing timescales could probe mass segregation deep in the potential of the $\mathrm{BH}$ (Miralda-Escudé \& Gould 2000). The vast majority of the stellar lenses are low-mass faint stars that are many magnitudes dimmer than the current detection limit. Microlensing can probe the statistical properties of these yet unobserved stars.

It is important to note that our quantitative results are subject to a number of approximations and uncertainties. For the sake of simplicity, we ignored the fact that the background stars may have a broad distribution of distances and hence different values for the Einstein radius. In addition, the model for the surface density and luminosity function of the distant background stars suffers from large uncertainties given the quality of present-day data. We did not attempt to deal in detail with the finite size of the stellar sources, which becomes relevant at high magnifications. We also ignored the contribution of blended light from the lensing star to the inferred flux from the source. However, this contribution is likely to be small for bright sources and low-mass lenses. Lastly, the semi-analytical formalism for calculating the magnification is not valid very near $\theta_{E}$ and so our quantitative predictions for $\left\langle N_{i}(>A)\right\rangle$ are less reliable for very high values of $A$. More precise calculations, for example by the ray-shooting method (Wambsganss 1997; Wambsganss \& Kubas 2000) will be required to improve the accuracy of the predictions.

Finally, we emphasize that our calculations can be applied to any extragalactic supermassive black hole. For example, the core of M87 harbors a black hole mass of $\sim 2 \times 10^{9} M_{\odot}$. The Einstein radius of this black hole for sources at infinity is $\sim 1^{\prime \prime}$, close to that of $\operatorname{Sgr} A^{\star}$, and there is no sign for obscuration by dust within its boundary. However, it is more likely that the sources will be stars in M87 a few kpc behind the black hole, for which $\theta_{E} \sim 0.03^{\prime \prime}$. For the higher black hole mass in M87, $\epsilon \sim 5 \times 10^{-10}$ and so microlensing events of stellar sources which are embedded in that galaxy will have durations comparable to those around $\operatorname{SgrA}^{\star}$ since $t \sim\left(\epsilon / M_{\bullet}\right)^{1 / 2}\left(D_{l} \theta_{e}\right)^{3 / 2}$, where $D_{l}$ is the distance from the observer to the BH. Since crowding and flux sensitivity severely limit the detection of individual stars at the distance of M87, one may search for microlensing of surface brightness fluctuations, the so-called pixel lensing (see, e.g. Gould 1996). The Next Generation Space Telescope (NGST; http://ngst.gsfc.nasa.gov/), with its sub-nJy sensitivity in 
the wavelength band of $\sim 1-3.5 \mu \mathrm{m}$ and its $\sim 0.06^{\prime \prime}$ resolution, is ideally suited for such a study.

We thank Joachim Wambsganss for communicating results from ray-shooting calculations

prior to publication (Wambsganss \& Kubas 2000) and an anonymous referee for useful comments. The KLF source model was revised following results communicated to us by J. Chanamé, A. Gould \& J. Miralda-Escudé prior to publication. AL thanks the Institute for Advanced Study for its kind hospitality during the initiation of this paper. This work was supported in part by NASA grants NAG 5-7768, 5-7039 and NSF grants AST-0071019, AST-0071019 (for AL).

\section{REFERENCES}

Alexander, T., 1999, ApJ, 527, 835

Alexander, T., \& Sternberg, A., 1999, ApJ, 520, 137

Bahcall, J. N., \& Wolf, R. A., 1977, ApJ, 216, 883

Blum R. D., Sellgren, K. \& DePoy, D. L., 1996, ApJ, 470, 864

Bolatto, A. D., \& Falco, E. E., 1994, ApJ, 436, 112

Chang, K. \& Refsdal, S., 1979, Nature, 282, 561 , 1984, A\&A, 132, 168

Davidge, T. J., Simons, D. A., Rigaut, F., Doyon, R., \& Crampton, D., 1997, AJ,114, 2586

Dominik, M., 1999, A\&A, 349,108

Eckart, A. \& Genzel, R., 1997, MNRAS, 284, 576

Gaudi, B. S., \& Gould, A., 1997, ApJ, 486, 85

Gaudi, B. S., Naber, R. M., \& Sackett, P. D. 1998, ApJ, 502, L33

Gaudi, B. S., \& Sackett, P. D., 2000, ApJ, 528, 56

Genzel, R., Eckart, A., Ott, T., \& Eisenhauer, F., 1997, MNRAS, 291 , 21

Genzel, R., Pichon, C., Eckart, A., Gerhard, O. E., \& Ott, T., 2000, MNRAS, 317, 418

Ghez, A. M., Klein, B. L., Morris, M., \& Becklin, E. E., 1998, ApJ, 509, 678

Gould, A., 1996, ApJ, 470, 201

Gould, A. \& Loeb, A., 1992, ApJ, 1992, 396, 104 
Griest, K., \& Safizadeh, N., 1998, ApJ, 500, 37

Holtzman, J. A., et al., 1998, AJ, 115, 1946

Kent, S. M., 1992, ApJ, 387, 181

Mao, S., \& Paczynski, B., 1991, ApJ, 374, L37

Miralda-Escudé, J., \& Gould, A., 2000, ApJ, submitted (astro-ph/0003269)

Ortiz, R., \& Lépine, J. R. D., 1993, A\&A, 279, 90

Paczynski, B., 1996, ARA\&A, 34, 419

Peale, S., 1997, Icarus 127, 269

Reid, M., 1993, ARA\&A, 31, 345

Schneider, P., Ehlers, J., \& Falco, E. E., 1992, Gravitational Lenses, (Springer: New York), pp. $313-320$

Tiede, G. P., Frogel, J. A., \& Terndrup, D. M., 1995, AJ, 110, 2788

Wainscoat R. J., Cohen, M., Volk, K., Walker, H. J., Schwartz, D. E., 1992, ApJSS, 83, 111

Wambsganss, J., 1997, MNRAS, 284, 172

Wambsganss, J., \& Kubas, D., 2000, in preparation

Wardle, M. \& Yusef-Zadeh, F., 1992, ApJ, 387, L65 


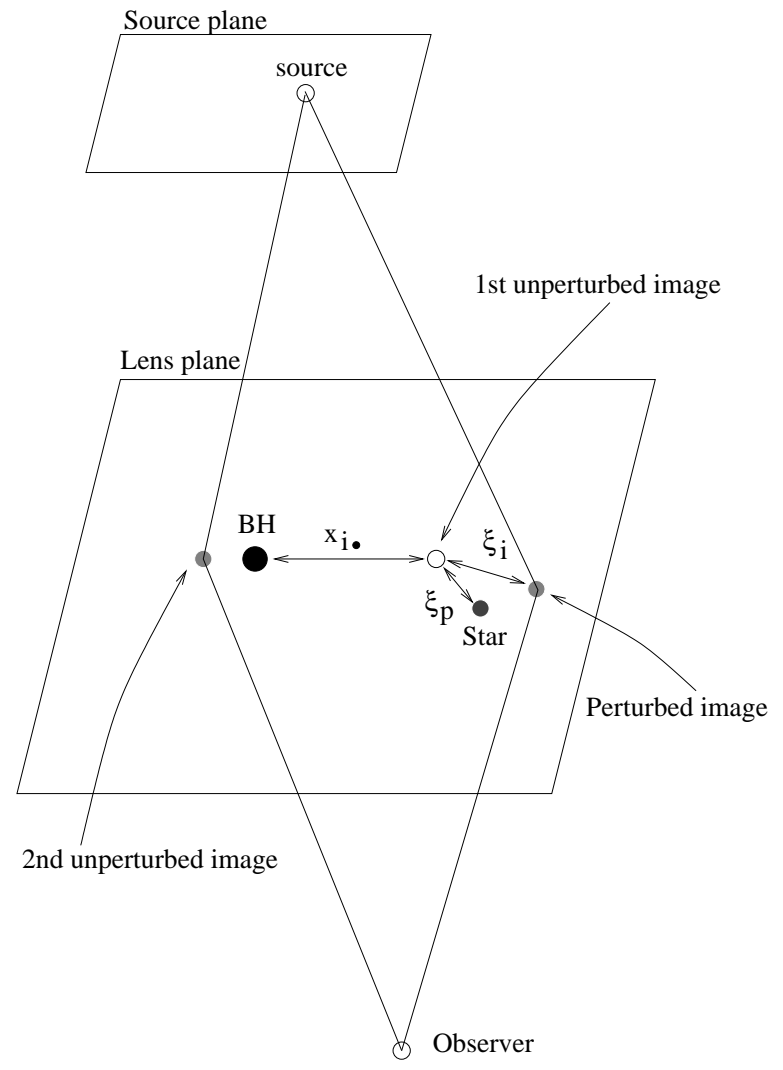

Fig. 1. - A sketch defining the notation used in this paper. The presence of a perturbing star at position $\boldsymbol{\xi}_{p}$ relative to the unperturbed image at $\boldsymbol{x}_{i}$ splits this image into 2 or 4 images at positions $\boldsymbol{\xi}_{i}$ relative to the unperturbed image. For clarity, only one of the multiple images due the the star is shown and the proportions are exaggerated. 

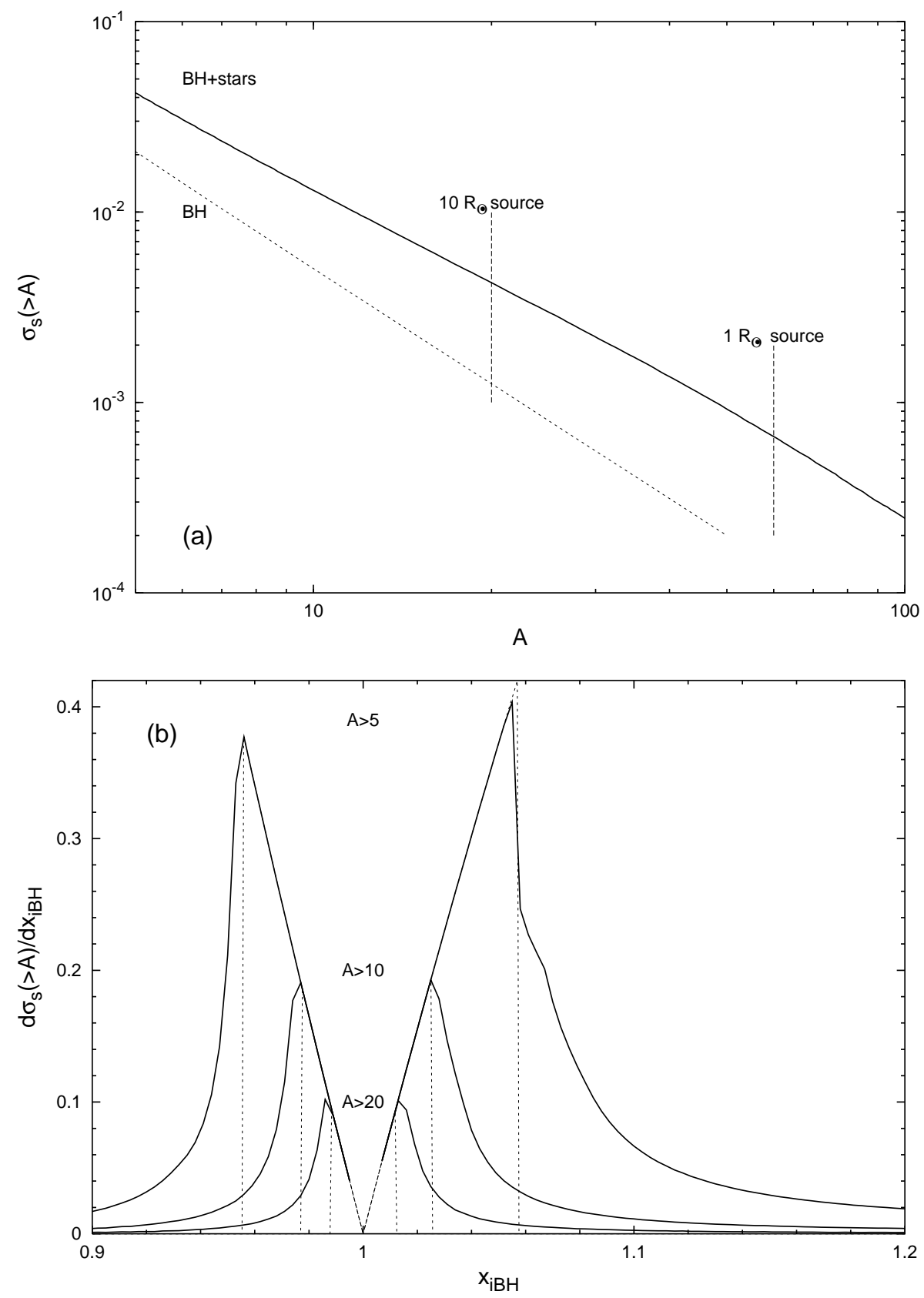

Fig. 2.- (a) The mean area in the source plane for magnification by more than $A$ by the $\mathrm{BH}$ alone, and by the $\mathrm{BH}$ and stars of an image in the inner $2 \theta_{E}$, for $\theta_{E}=\theta_{\infty}$ and the stellar lens surface density model of equation (11) with $\alpha=3 / 2$ (see text for scaling to arbitrary $\theta_{E}$ ). The two vertical hash marks roughly indicate the maximal magnification possible for extended sources, a $1 R_{\odot}$ and a $10 R_{\odot}$ star at a distance of $2 R_{0}$. (b) The differential source area per annulus in the lens plane that is magnified by more than $A$. The case of a $\mathrm{BH}$ and stars (full lines) is compared to the contribution from the $\mathrm{BH}$ alone (dashed lines) for three threshold values of $A$. 

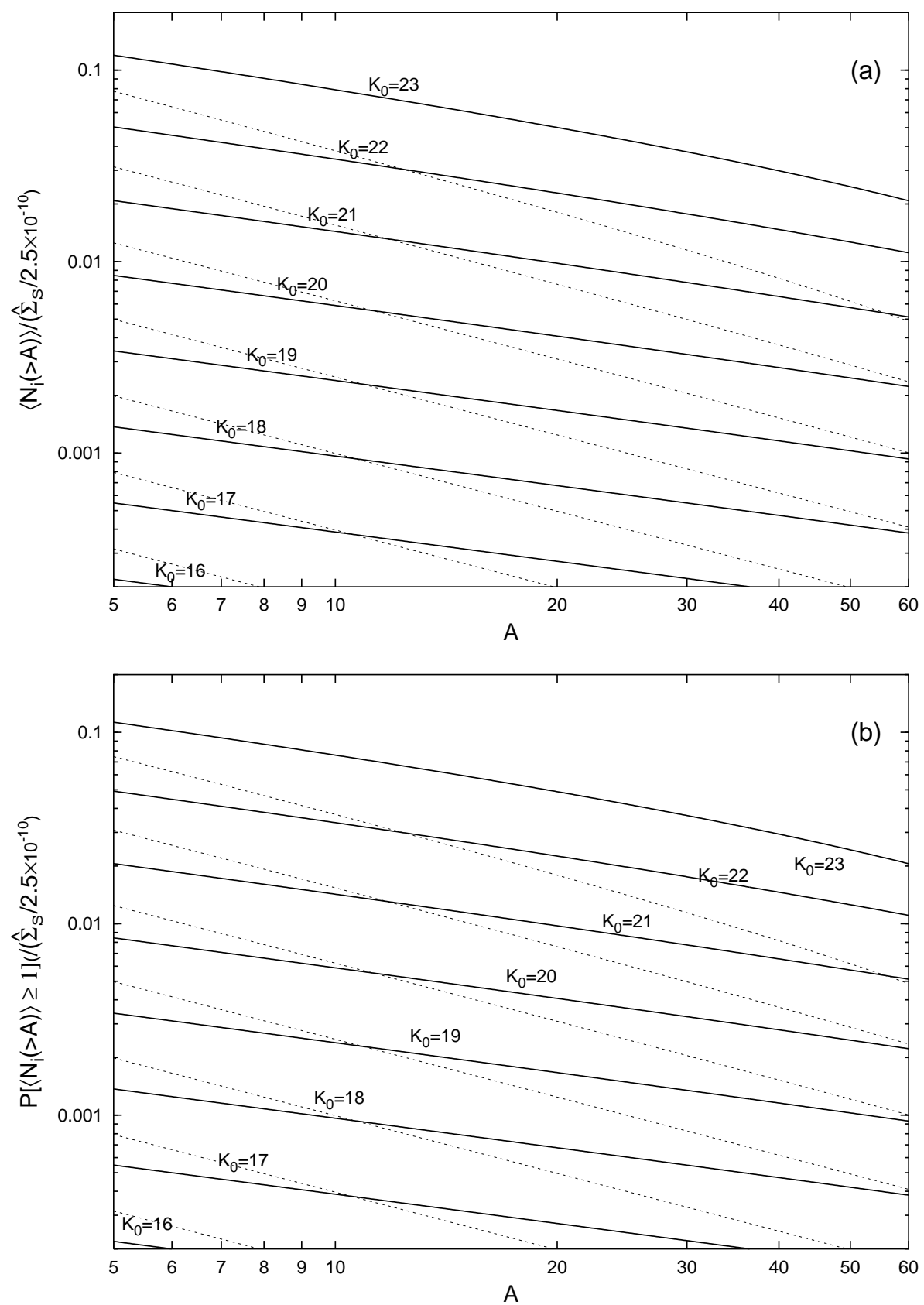

Fig. 3.- (a) The average number of lensed images magnified by more than $A$ that will be observed in the inner $2 \theta_{E}$ with a limiting $K$-band magnitude $K_{0}$, for $D_{s}=2 R_{0}$ and the stellar lens surface density model of equation (11) with $\alpha=3 / 2$. (b) The fraction of time that at least one lensed image magnified by more than $A$, will be observed in the inner $2 \theta_{E}$ with a limiting $K$-band magnitude $K_{0}$ $\left(P \ll 1\right.$ scales linearly with $\widehat{\Sigma}_{s}$ ). The labeled solid lines are for the BH and stars and the unlabeled dotted lines, which follow the same $K_{0}$ order, are for the $\mathrm{BH}$ alone. 


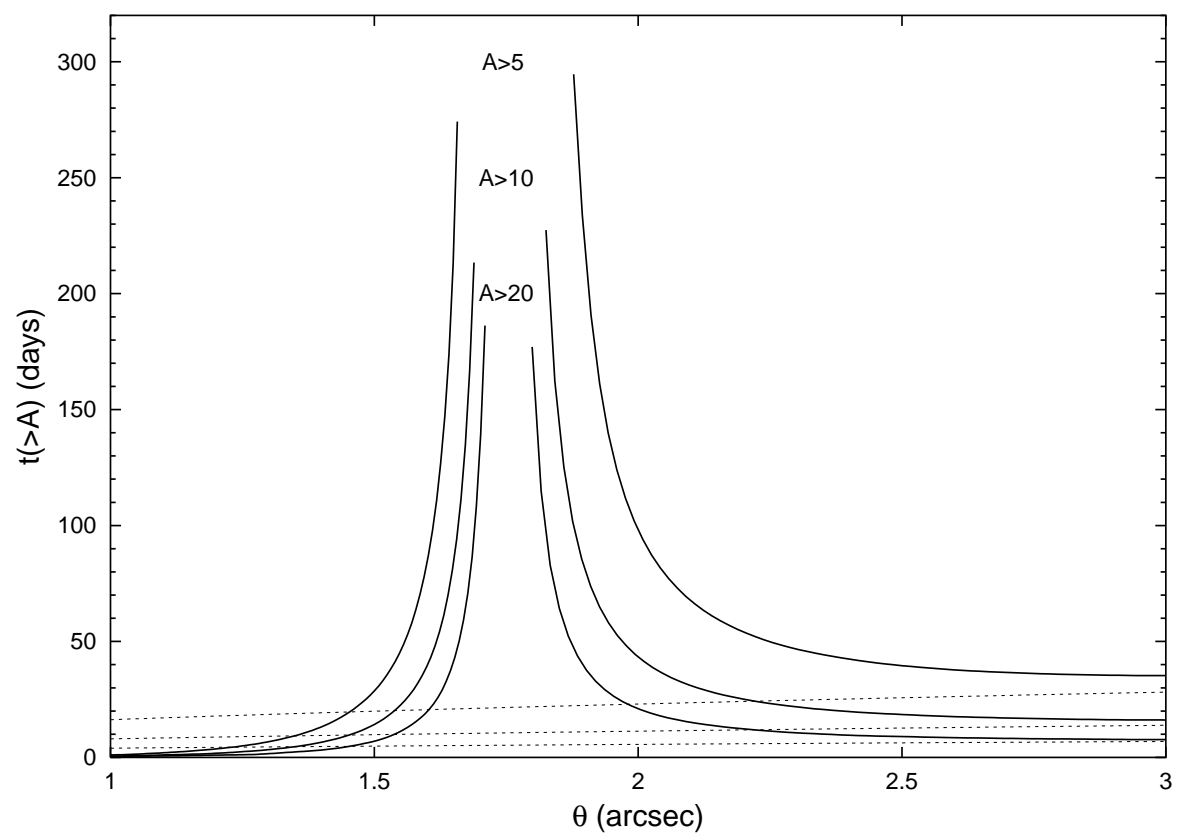

Fig. 4. - The typical duration of a microlensing event for a stationary source at infinity by a solar mass lens (assuming the mean rms velocity of equation 15]), as a function of angular separation $\theta=x_{i} \theta_{\infty}$ from $\operatorname{Sgr}^{\star}$. Curves are shown for different magnification values $A$, comparing stars in the shear field of the BH (full lines) with isolated stars moving at the same velocity (dashed lines). 